\title{
Flow field of impinging sweeping jets
}

\author{
G. Paolillo $^{1 *}$, C. S. Greco ${ }^{1}$, G. Cardone ${ }^{1}$ and T. Astarita ${ }^{1}$ \\ ${ }^{1}$ University of Naples “Federico II”, Department of Industrial Engineering, Naples 80125, Italy \\ *gerardo.paolillo@unina.it
}

Sweeping jets are oscillating jets generated by fluidic oscillators, i.e., devices designed to produce an oscillation of the flow without the use of any moving parts (Raghu, 2013). A typical configuration of such devices consists of an expansion chamber connected to a high-pressure supply via a converging nozzle and provided with feedback channels. The oscillating motion in the expansion chamber is triggered by an inherent flow instability and sustained by the flow rate across the feedback channels. Recently, sweeping jets have been studied in flow control applications for noise reduction, separation and circulation control over airfoils, control of resonant cavity oscillations and deflection of jets. The advantageous features of fluidic actuators, among which are the wide range of operating frequencies (up to $\mathrm{kHz}$ with meso-scale) and the distributed momentum addition, have also stimulated an increasing interest in their application to electronics cooling. Several recent studies on the convective heat transfer from impinging sweeping jets (e.g., Hossain et al., 2018; Park et al., 2018) have shown that, compared to conventional round jets, they offer higher cooling rates with better uniformity at least for small jet-to-plate spacings.

In the present study, the flow field of impinging sweeping jets is experimentally investigated using particle image velocimetry (PIV) in order to provide insight into their heat transfer capabilities. The investigated sweeping jets are generated by different configurations of fluidic oscillators fabricated with a 3D printer. The geometry of such devices is schematically displayed in Figure $1 \mathrm{a}$. The width of the exit throat section $w$ is $10 \mathrm{~mm}$ and it is equal to the depth of the sweeping nozzle (the exit throat nozzle is square). The length of the expansion chamber $L_{f}$ is varied from one device to another; specifically, three different values of $L_{f}$ are investigated: $L_{f} / w=2.5,3.5,4.5$. The fluidic oscillator with the longest expansion chamber $\left(L_{f}=4.5 w\right)$ is also provided with tapped holes on its shortest sides, which culminate into the feedback channels. Fastening a screw in these holes allows to change the minimum passage area of the feedback channels and correspondingly the head loss across them. Such an expedient is used to vary the sweeping jet's Strouhal number, which was observed to be constant for a fixed geometry when varying the Reynolds number. The oscillating frequency of the sweeping jet is determined by recording the instantaneous head loss across one feedback channel (the pressure holes used for the differential pressure measurement are also illustrated in Figure 1 1 ). The experimental setup for PIV measurements is shown in Figure $1 \mathrm{~b}$. The impingement distance is varied by moving away the plate via a micrometric translational stage; five different spacings are investigated: $H / w=2,4,6,8,10$. The mass flow rate is measured by means of a flow meter and the Reynolds number $R e_{w}$, based on the square exit section edge $w$, is fixed to $1.7 \times 10^{4}$.
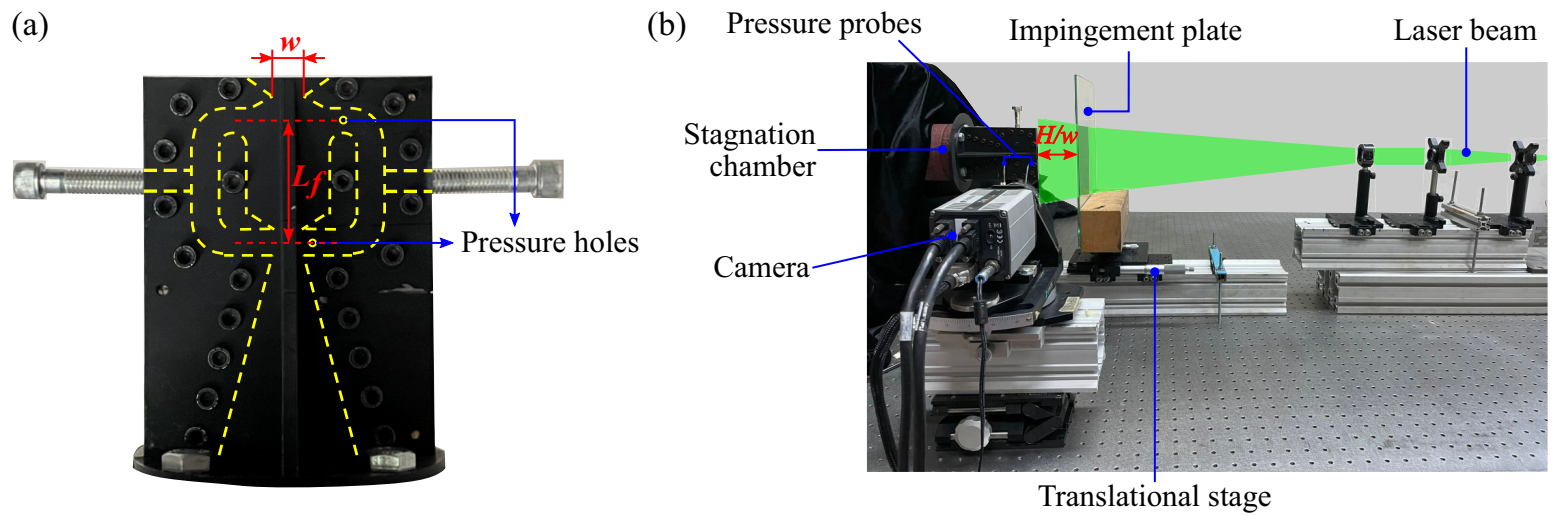

Figure 1: (a) Schematic of the internal geometry of the fluidic oscillators investigated in the present study and (b) experimental setup for the planar PIV measurements. 

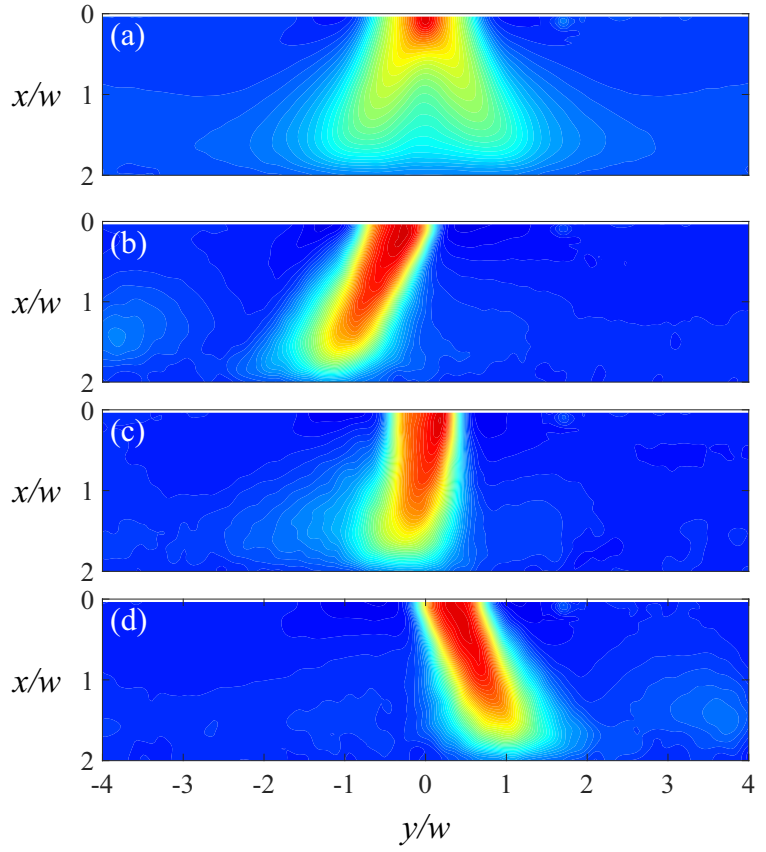
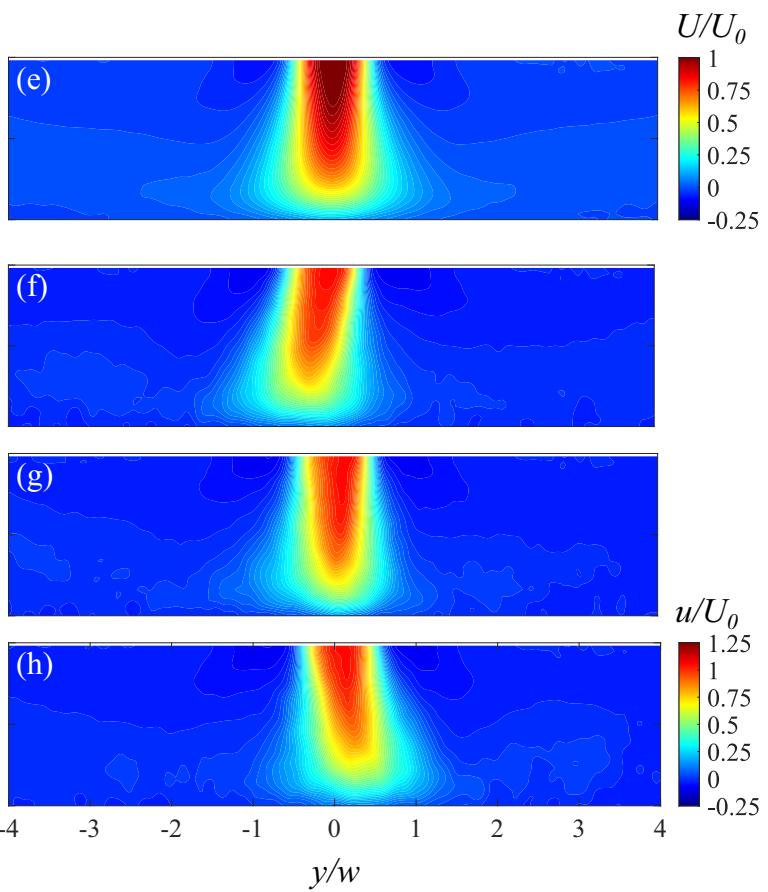

Figure 2: Streamwise velocity field of the impinging sweeping jet issuing from the fluidic oscillator with $L_{f} / w=4.5$ with open (a-d) and closed (e-h) feedback channels: (a, e) time average, (b-d,f-h) phase averages for different phases. $U_{0}$ is the area-averaged jet exit velocity. $R e_{w}=1.7 \times 10^{4}, H / w=2$.

For lengths of the expansion chamber smaller than $4.5 \mathrm{w}$, a coherently organized oscillating motion is not observed; conversely, the jet resembles more a conventional round jet. This is in agreement with the previous findings of the numerical work of Seo et al. (2018). Figure 2 shows the effects of reducing the minimum passage area of the feedback channels on the impinging flow field. While the oscillating frequency of the jet is observed to increase when closing the feedback channels, the amplitude of the jet oscillation is strongly reduced, as visible in the phase-averaged fields. As a consequence, the sweeping jet obtained with open feedback channels (Figures $2 \mathrm{a}$-d) presents, in the time-averaged field, a larger jet width, although this comes with lower momentum in the jet core. A more uniform and wider distribution of the heat transfer rate is thus expected for this case. In the present experiments, the phase-averaging is performed by calculating the phase angle from the instantaneous differential pressure signal recorded simultaneously with the particle images. A frequency jitter is indeed noticed, which prevents phase-averaging at one fixed frequency. However, the characteristic oscillating modes of the sweeping jet can be determined via proper orthogonal decomposition (POD), which also sheds light on the dynamics of structures with smaller scales. The turbulence statistics are presented in terms of a triple decomposition of the velocity field, thus separating the quasi-periodic coherent fluctuation from the random turbulent one.

\section{References}

Hossain MA, Prenter R, Lundgreen RK, Ameri A, Gregory JW, and Bons JP (2018) Experimental and numerical investigation of sweeping jet film cooling. Journal of Turbomachinery 140

Park T, Kara K, and Kim D (2018) Flow structure and heat transfer of a sweeping jet impinging on a flat wall. International Journal of Heat and Mass Transfer 124:920-928

Raghu S (2013) Fluidic oscillators for flow control. Experiments in Fluids 54:1-11

Seo J, Zhu C, and Mittal R (2018) Flow physics and frequency scaling of sweeping jet fluidic oscillators. AIAA Journal 56:2208-2219 